\title{
RATIONAL TORSION POINTS ON JACOBIANS OF MODULAR CURVES
}

\author{
HWAJONG YOO
}

\begin{abstract}
Let $p$ be a prime greater than 3. Consider the modular curve $X_{0}(3 p)$ over $\mathbb{Q}$ and its Jacobian variety $J_{0}(3 p)$ over $\mathbb{Q}$. Let $\mathcal{T}(3 p)$ and $\mathcal{C}(3 p)$ be the group of rational torsion points on $J_{0}(3 p)$ and the cuspidal group of $J_{0}(3 p)$, respectively. We prove that the 3 -primary subgroups of $\mathcal{T}(3 p)$ and $\mathcal{C}(3 p)$ coincide unless $p \equiv 1(\bmod 9)$ and $3^{\frac{p-1}{3}} \equiv 1(\bmod p)$.
\end{abstract}

\section{CONTENTS}

1. Introduction

2. Eisenstein ideals of level $p q$

3. Case where $\ell$ does not divide $p q \quad 3$

4. Case where $\ell=p$ or $\ell=q \quad 4$

References

\section{INTRODUCTION}

Let $N$ be a square-free integer. Consider the modular curve $X_{0}(N)$ and its Jacobian variety $J_{0}(N)=\operatorname{Pic}^{0}\left(X_{0}(N)\right)$. Let $\mathcal{T}(N)$ denote the group of rational torsion points on $J_{0}(N)$ and let $\mathcal{C}(N)$ denote the cuspidal group of $J_{0}(N)$. By Manin and Drinfeld [2,3], we have $\mathcal{C}(N) \subseteq \mathcal{T}(N)$ and they are both finite abelian groups.

When $N$ is prime, $\mathrm{Ogg}$ conjectured that $\mathcal{T}(N)=\mathcal{C}(N)$ [5, Conjecture 2]. In his article [4], Mazur proved this conjecture by studying the Eisenstein ideal of level $N$. Recently, Ohta proved a generalization of the result of Mazur [6]. More precisely, he proved the following.

Theorem 1.1 (Ohta). For a prime $\ell \geq 5$, we have $\mathcal{T}(N)\left[\ell^{\infty}\right]=\mathcal{C}(N)\left[\ell^{\infty}\right]$. Moreover, if 3 does not divide $N$, then $\mathcal{T}(N)\left[3^{\infty}\right]=\mathcal{C}(N)\left[3^{\infty}\right]$.

(For a finite abelian group $A, A\left[\ell^{\infty}\right]$ denotes the $\ell$-primary subgroup of $A$.)

We briefly sketch the proof of this theorem. Let $T_{r}$ (resp. $U_{p}$ and $w_{p}$ ) denote the $r^{\text {th }}$ Hecke operator (resp. the $p^{\text {th }}$ Hecke operator and the Atkin-Lehner operator with respect to $p$ ) acting on $J_{0}(N)$ for a prime $r$ not dividing $N$ (resp. a prime divisor $p$ of $N$ ). Let $\mathbb{T}(N)$ (resp. $\left.\mathbb{T}(N)^{\prime}\right)$ be the $\mathbb{Z}$-subalgebra of $\operatorname{End}\left(J_{0}(N)\right)$ generated by $T_{r}$ 's and $U_{p}$ 's (resp. $T_{r}$ 's and $w_{p}$ 's) for primes $r \nmid N$ and $p \mid N$. Let

$$
\mathcal{I}_{0}:=\left(T_{r}-r-1: \text { for primes } r \nmid N\right)
$$

be the (minimal) Eisenstein ideal of $\mathbb{T}(N)$ (or $\mathbb{T}(N)^{\prime}$ ). Then, $\mathcal{I}_{0}$ annihilates $\mathcal{T}(N)$ and $\mathcal{C}(N)$ by the EichlerShimura relation. Thus, $\mathcal{T}(N)\left[\ell^{\infty}\right]$ is a module over $\mathbb{T}(N)_{\ell} / \mathcal{I}_{0}$ (or $\mathbb{T}(N)_{\ell}^{\prime} / \mathcal{I}_{0}$ ), where $\mathbb{T}(N)_{\ell}:=\mathbb{T}(N) \otimes_{\mathbb{Z}} \mathbb{Z}_{\ell}$. Note that since $w_{p}^{2}=1$, for a prime $\ell \geq 3$ we have the following decomposition:

$$
\mathbb{T}(N)_{\ell}^{\prime} / \mathcal{I}_{0}=\prod_{M \mid N, M \neq N} \mathbb{T}(N)_{\ell}^{\prime} / \mathcal{I}_{M},
$$

where $\mathcal{I}_{M}:=\left(w_{p}-1, w_{q}+1, \mathcal{I}_{0}:\right.$ for primes $p \mid M$ and $\left.q \mid N / M\right)$. Thus, we have

$$
\mathcal{T}(N)\left[\ell^{\infty}\right]=\oplus \mathcal{T}(N)\left[\ell^{\infty}\right]\left[\mathcal{I}_{M}\right] \quad \text { and } \quad \mathcal{C}(N)\left[\ell^{\infty}\right]=\oplus \mathcal{C}(N)\left[\ell^{\infty}\right]\left[\mathcal{I}_{M}\right]
$$

2010 Mathematics Subject Classification. 11G10, 11G18, $14 \mathrm{G} 05$.

Key words and phrases. Rational points, modular curves, Eisenstein ideals.

This work was supported by IBS-R003-G1. 
Finally, he proved that $\mathcal{T}(N)\left[\ell^{\infty}\right]\left[\mathcal{I}_{M}\right]=\mathcal{C}(N)\left[\ell^{\infty}\right]\left[\mathcal{I}_{M}\right]$ by computing the index of $\mathcal{I}_{M}$ (up to 2-primary parts).

In this paper, we discuss the case where $N=p q$ for two distinct primes $p$ and $q$. In contrast to the discussion above, we use $\mathbb{T}(p q)$ instead of $\mathbb{T}(p q)^{\prime}$ and hence the corresponding decomposition of $\mathbb{T}(p q) / \mathcal{I}_{0}$ as above does not always exist. (However, other computations are relatively easier than the method by Ohta.) When $\ell$ satisfies some conditions, we get the similar decomposition of the quotient ring $\mathbb{T}(p q) / \mathcal{I}_{0}$ and we can prove the following.

Theorem 1.2 (Main Theorem). For a prime $\ell$ not dividing $2 p q \operatorname{gcd}(p-1, q-1)$, we have $\mathcal{T}(p q)\left[\ell^{\infty}\right]=\mathcal{C}(p q)\left[\ell^{\infty}\right]$. Moreover, $\mathcal{T}(p q)\left[p^{\infty}\right]=\mathcal{C}(p q)\left[p^{\infty}\right]$ if one of the following holds:

(1) $p \geq 5$ and $\left\{\begin{array}{l}\text { either } q \not \equiv 1(\bmod p) \text { or } \\ q \equiv 1(\bmod p) \text { and } p^{\frac{q-1}{p}} \not \equiv 1(\bmod q) .\end{array}\right.$
(2) $p=3$ and $\left\{\begin{array}{l}\text { either } q \not \equiv 1(\bmod 9) \text { or } \\ q \equiv 1(\bmod 9) \text { and } 3^{\frac{q-1}{3}} \not \equiv 1(\bmod q) .\end{array}\right.$

Note that most cases are special ones of Theorem 1.1. The new result is as follows:

Theorem 1.3. Let $p$ be a prime greater than 3. Assume that either $p \not \equiv 1(\bmod 9)$ or $3^{\frac{p-1}{3}} \not \equiv 1(\bmod p)$. Then, we get

$$
\mathcal{T}(3 p)\left[3^{\infty}\right]=\mathcal{C}(3 p)\left[3^{\infty}\right]
$$

1.1. Notation. For $x=a / b \in \mathbb{Q}$, we denote by $\operatorname{num}(x)$ the numerator of $x$, i.e.,

$$
\operatorname{num}(x):=\frac{a}{(a, b)} .
$$

From now on, we denote by $\ell^{\alpha}:=\ell^{\alpha(p, q, \ell)}\left(\right.$ resp. $\left.\ell^{\beta}:=\ell^{\beta(p, q, \ell)}\right)$ the exact power of $\ell$ dividing

$$
M_{p}:=\operatorname{num}\left(\frac{(p-1)\left(q^{2}-1\right)}{3}\right)\left(\text { resp. num }\left(M_{q}:=\frac{\left(p^{2}-1\right)(q-1)}{3}\right)\right) .
$$

\section{EISENSTEIN IDEALS OF LEVEL $p q$}

Throughout this section, we fix two distinct primes $p$ and $q$; and $\ell$ denotes a prime not dividing $2 p q(q-1)$. Let $\mathbb{T}:=\mathbb{T}(p q)$ and $\mathbb{T}_{\ell}:=\mathbb{T}(p q) \otimes_{\mathbb{Z}} \mathbb{Z}_{\ell}$. We say that an ideal of $\mathbb{T}$ is Eisenstein if it contains

$$
\mathcal{I}_{0}:=\left(T_{r}-r-1: \text { for primes } r \nmid p q\right) .
$$

Definition 2.1. We define Eisenstein ideals as follows:

$$
\begin{gathered}
\mathcal{I}_{1}:=\left(U_{p}-1, U_{q}-1, \mathcal{I}_{0}\right) \\
\mathcal{I}_{2}:=\left(U_{p}-1, U_{q}-q, \mathcal{I}_{0}\right) \quad \text { and } \quad \mathcal{I}_{3}:=\left(U_{p}-p, U_{q}-1, \mathcal{I}_{0}\right) .
\end{gathered}
$$

Moreover, we set $\mathfrak{m}_{i}:=\left(\ell, \mathcal{I}_{i}\right)$. They are all possible Eisenstein maximal ideals in $\mathbb{T}_{\ell}$ by the result in [9, $\left.\S 2\right]$. For ease of notation, we set $\mathbb{T}_{i}:=\mathbb{T}_{\mathfrak{m}_{i}}=\lim _{\leftarrow n} \mathbb{T} / \mathfrak{m}_{i}^{n}$.

Since $\mathbb{T}_{\ell}$ is a semi-local ring, we have

$$
\mathbb{T}_{\ell}=\prod_{\ell \in \mathfrak{m} \text { maximal }} \mathbb{T}_{\mathfrak{m}}
$$

Using the above description of Eisenstein maximal ideals, we prove the following:

Theorem 2.2. The quotient $\mathbb{T}_{\ell} / \mathcal{I}_{0}$ is isomorphic to $\mathbb{T}_{\ell} / \mathcal{I}_{2} \times \mathbb{T}_{\ell} / \mathcal{I}_{3}$.

This theorem is crucial to deduce our main theorem. In general, the author expects that $\mathbb{T}_{\ell} / \mathcal{I}_{0}$ should be isomorphic to

$$
\left\{(x, y, z) \in \mathbb{T}_{\ell} / \mathcal{I}_{1} \times \mathbb{T}_{\ell} / \mathcal{I}_{2} \times \mathbb{T}_{\ell} / \mathcal{I}_{3}: x \equiv y(\bmod p-1) \text { and } x \equiv z(\bmod q-1)\right\} .
$$

Before proving the theorem above, we need several lemmas.

Lemma 2.3. We have $\left(U_{p}-1\right)\left(U_{p}+1\right) \in \mathcal{I}_{0} \mathbb{T}_{\ell}$. 


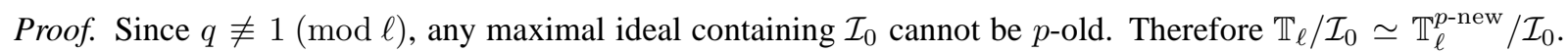
Since $U_{p}^{2}=1$ in $\mathbb{T}_{\ell}^{p \text {-new }}$, the result follows.

Lemma 2.4. Suppose that $\mathfrak{m}_{2}$ is maximal. Then, we have

$$
\mathbb{T}_{2} / \mathcal{I}_{0}=\mathbb{T}_{2} / \mathcal{I}_{2} \simeq \mathbb{T}_{\ell} / \mathcal{I}_{2}
$$

If $\mathfrak{m}_{1}$ is maximal, then $p \equiv 1(\bmod \ell)$ and hence $\mathfrak{m}_{1}=\mathfrak{m}_{3} ;$ moreover, we have $\mathbb{T}_{1} / \mathcal{I}_{0}=\mathbb{T}_{3} / \mathcal{I}_{0} \simeq \mathbb{T}_{\ell} / \mathcal{I}_{3}$. If $p \not \equiv 1(\bmod \ell)$, then $\mathfrak{m}_{1}$ is not maximal and $\mathbb{T}_{3} / \mathcal{I}_{0} \simeq \mathbb{T}_{\ell} / \mathcal{I}_{3}$.

Proof. Suppose that $\mathfrak{m}_{2}$ is maximal. Since $U_{p}-1 \in \mathfrak{m}_{2}$ and $\ell$ is odd, $U_{p}+1 \notin \mathfrak{m}_{2}$ and hence it is a unit in $\mathbb{T}_{2}$. By the lemma above, $\left(U_{p}-1\right)\left(U_{p}+1\right) \in \mathcal{I}_{0} \mathbb{T}_{\ell}$ and hence $U_{p}-1 \in \mathcal{I}_{0} \mathbb{T}_{2}$. Similarly, we have $U_{q}-q \in \mathcal{I}_{0} \mathbb{T}_{2}$ because $q \not \equiv 1(\bmod \ell)$ and $\left(U_{q}-1\right)\left(U_{q}-q\right) \in \mathcal{I}_{0} \mathbb{T}_{2}$ by the following lemma. Thus, we have $\mathbb{T}_{2} / \mathcal{I}_{0}=\mathbb{T}_{2} / \mathcal{I}_{2}$. Since the index of $\mathcal{I}_{2}$ in $\mathbb{T}$ is finite (cf. [7, Lemma 3.1]), we have $\mathfrak{m}_{2}^{n} \subseteq \mathcal{I}_{2}$ for large enough $n$. Therefore $\mathbb{T}_{\ell} /\left(\mathfrak{m}_{2}^{n}, \mathcal{I}_{2}\right) \simeq \mathbb{T}_{\ell} / \mathcal{I}_{2}$ and hence $\mathbb{T}_{2} / \mathcal{I}_{2} \simeq \mathbb{T}_{\ell} / \mathcal{I}_{2}$

If $\mathfrak{m}_{1}$ is maximal, the index of $\mathcal{I}_{1}$ in $\mathbb{T}$ is divisible by $\ell$. By [9, Theorem 1.4], it is $\operatorname{num}\left(\frac{(p-1)(q-1)}{3}\right)$ up to powers of 2 and hence $p \equiv 1(\bmod \ell)$.

Assume that $p \equiv 1(\bmod \ell)$. Let $\alpha$ be the number in $\S 1$. Since $\ell$ does not divide $(p+1)(q-1)$, $\ell^{\alpha}$ divides $(p-1)$. Note that the index of $\mathcal{I}_{3}$ in $\mathbb{T}_{\ell}$ is equal to $\ell^{\alpha}$ (cf. [9, Theorem 1.4]) and hence $\mathcal{I}_{3} \mathbb{T}_{\ell}$ contains $p-1$. Thus, $U_{p}-1=\left(U_{p}-p\right)+(p-1) \in \mathcal{I}_{3} \mathbb{T}_{\ell}$. In other words, $\mathcal{I}_{1} \mathbb{T}_{\ell} \subseteq \mathcal{I}_{3} \mathbb{T}_{\ell}$. Similarly, we have $\mathcal{I}_{3} \mathbb{T}_{\ell} \subseteq \mathcal{I}_{1} \mathbb{T}_{\ell}$. Therefore we have $\mathcal{I}_{1} \mathbb{T}_{\ell}=\mathcal{I}_{3} \mathbb{T}_{\ell}$. By the same argument as above, $\mathcal{I}_{0} \mathbb{T}_{3}$ contains $U_{p}-1$ and $\left(U_{q}-1\right)\left(U_{q}-q\right)$. Since $q \not \equiv 1(\bmod \ell)$ and $U_{q}-1 \in \mathfrak{m}_{3}$, we have $U_{q}-q \notin \mathfrak{m}_{3}$ and hence $\mathbb{T}_{3} / \mathcal{I}_{0}=\mathbb{T}_{3} / \mathcal{I}_{3}$. By the same argument as above, we get $\mathbb{T}_{3} / \mathcal{I}_{3} \simeq \mathbb{T}_{\ell} / \mathcal{I}_{3}$.

If $p \not \equiv 1(\bmod \ell)$, then $\mathfrak{m}_{3}$ is neither $p$-old nor $q$-old. If $p \not \equiv-1(\bmod \ell)$, then $\mathfrak{m}_{3}$ is not maximal. Thus, we have $\mathbb{T}_{\ell} / \mathcal{I}_{3}=\mathbb{T}_{3} / \mathcal{I}_{0}=0$. If $p \equiv-1(\bmod \ell)$, then the result follows by [8, Proposition 2.3].

Lemma 2.5. Let $I:=\left(U_{p}-1, \mathcal{I}_{0}\right) \subseteq \mathbb{T}_{\ell}$. Then, we get $\left(U_{q}-1\right)\left(U_{q}-q\right) \in I$.

Proof. We closely follow the argument in [4, §II. 5].

Let $f(z):=\sum_{n \geq 1}\left(T_{n} \bmod I\right) x^{n}$ be the Fourier expansion (at $\infty$ ) of a cusp form of weight 2 and level $p q$ over $\mathbb{T}_{\ell} / I$, where $x=e^{2 \pi i z}$. Let $E:=E_{p, p q}$ be an Eisenstein series of weight 2 and level $p q$ in $[7, \S 2.3]$. Note that

$$
(f-E)(z) \equiv\left(U_{q}-q\right) \sum_{n \geq 1} a_{n} x^{q n}(\bmod I),
$$

where $a_{p}=1$ and $a_{r}=1+r$ for all primes $r \neq p q$; and $a_{q}=U_{q}+q$. If $U_{q}-q \notin I$, then by Ohta [6, Lemma 2.1.1], there is a cusp form $g(z)=\sum_{n \geq 1} b_{n} x^{n}$ of weight 2 and level $p$ such that

$$
(f-E)(z) \equiv\left(U_{q}-q\right) \sum_{n \geq 1} a_{n} x^{q n} \equiv\left(U_{q}-q\right) g(q z)(\bmod I) .
$$

Therefore $p \equiv 1(\bmod \ell)$ and $b_{r} \equiv 1+r\left(\bmod I^{\prime}\right)$ for primes $r \neq p$, where $I^{\prime}$ is the Eisenstein ideal of level $p$. Thus, we have $\left(U_{q}-q\right)\left(a_{q}-b_{q}\right) \equiv\left(U_{q}-q\right)\left(U_{q}-1\right) \in I$.

Now, we are ready to prove Theorem 2.2.

Proof of Theorem 2.2. If $p \equiv 1(\bmod \ell)$, then $\mathfrak{m}_{1}=\mathfrak{m}_{3}$. Otherwise $\mathfrak{m}_{1}$ is not maximal. Therefore, we have

$$
\mathbb{T}_{\ell} / \mathcal{I}_{0} \simeq \mathbb{T}_{2} / \mathcal{I}_{0} \times \mathbb{T}_{3} / \mathcal{I}_{0}=\mathbb{T}_{2} / \mathcal{I}_{2} \times \mathbb{T}_{3} / \mathcal{I}_{3} \simeq \mathbb{T}_{\ell} / \mathcal{I}_{2} \times \mathbb{T}_{\ell} / \mathcal{I}_{3}
$$

\section{CASE WHERE $\ell$ DOES NOT DIVIDE $p q$}

From now on, let $\mathcal{C}:=\mathcal{C}(p q)$ and $\mathcal{T}:=\mathcal{T}(p q)$ be the cuspidal group of $J_{0}(p q)$ and the group of rational torsion points on $J_{0}(p q)$, respectively. For a prime $r$ and a finite abelian group $A$, we denote by $A\left[r^{\infty}\right]$ the $r$-primary subgroup of $A$. In this section, we prove the following theorem.

Theorem 3.1. For a prime $\ell$ not dividing $2 p q(q-1)$, we have $\mathcal{T}\left[\ell^{\infty}\right]=\mathcal{C}\left[\ell^{\infty}\right]$. 
Before proving this theorem, we introduce some cuspidal divisors.

Let $P_{n}$ be the cusp of $X_{0}(p q)$ corresponding to $1 / n \in \mathbb{P}^{1}(\mathbb{Q})$. Let $C_{p}:=P_{1}-P_{p}$ and $C_{q}:=P_{1}-P_{q}$ denote the cuspidal divisors in $\mathcal{C}$. Let $M_{p}=\ell^{\alpha} \times x$ and $M_{q}=\ell^{\beta} \times y$ as in $\S 1.1$. (Thus, we have $(\ell, x y)=1$.) We define

$$
D_{p}:=x C_{p} \quad \text { and } \quad D_{q}:=y C_{q} .
$$

Then, $\left\langle D_{p}\right\rangle$ (resp. $\left\langle D_{q}\right\rangle$ ) is a free module of rank 1 over $\mathbb{T}_{\ell} / \mathcal{I}_{2} \simeq \mathbb{Z} / \ell^{\alpha} \mathbb{Z}$ (resp. $\mathbb{T}_{\ell} / \mathcal{I}_{3} \simeq \mathbb{Z} / \ell^{\beta} \mathbb{Z}$ ) (cf. [9, Theorem $1.4])$.

Now we prove the Theorem above.

Proof of Theorem 3.1. By the Eichler-Shimura relation, $\mathcal{T}\left[\ell^{\infty}\right]$ is a module over $\mathbb{T}_{\ell} / \mathcal{I}_{0}$. Therefore $\mathcal{T}\left[\ell^{\infty}\right]$ decomposes into $\mathcal{T}\left[\ell^{\infty}\right]\left[\mathcal{I}_{2}\right] \times \mathcal{T}\left[\ell^{\infty}\right]\left[\mathcal{I}_{3}\right]$ by Theorem 2.2. Hence it suffices to show that $\mathcal{T}\left[\ell^{\infty}\right]\left[\mathcal{I}_{2}\right]=\left\langle D_{p}\right\rangle$ and $\mathcal{T}\left[\ell^{\infty}\right]\left[\mathcal{I}_{3}\right]=\left\langle D_{q}\right\rangle$.

If $\alpha=0$, then $\mathbb{T}_{\ell} / \mathcal{I}_{2}=0$ and hence $\mathcal{T}\left[\ell^{\infty}\right]\left[\mathcal{I}_{2}\right]=\left\langle D_{p}\right\rangle=0$. Thus, we may assume that $\alpha \geq 1$. Note that

$$
\mathcal{T}\left[\ell^{\infty}\right]\left[\mathcal{I}_{2}\right] \simeq \prod_{i=1}^{t} \mathbb{Z} / \ell^{a_{i}} \mathbb{Z}
$$

where $1 \leq a_{i} \leq \alpha$ because $\mathbb{T}_{\ell} / \mathcal{I}_{2} \simeq \mathbb{Z} / \ell^{\alpha} \mathbb{Z}$ (and $\mathcal{T}$ is finite). Since $D_{p} \in \mathcal{T}\left[\ell^{\infty}\right]$, we have $\left\langle D_{p}\right\rangle \subseteq \mathcal{T}\left[\ell^{\infty}\right]\left[\mathcal{I}_{2}\right]$ and hence $t \geq 1$; and $\mathcal{T}\left[\ell^{\infty}\right]\left[\ell, \mathcal{I}_{2}\right] \simeq(\mathbb{Z} / \ell \mathbb{Z})^{\oplus t} \subseteq J_{0}(N)\left[\mathfrak{m}_{2}\right]$. By the same argument in [4, §II, Corollary 14.8] (cf. [7, Theorem 4.2]), we have $t=1$ and $\mathcal{T}\left[\ell^{\infty}\right]\left[\mathcal{I}_{2}\right]=\left\langle D_{p}\right\rangle$. By symmetry, $\mathcal{T}\left[\ell^{\infty}\right]\left[\mathcal{I}_{3}\right]=\left\langle D_{q}\right\rangle$ and the result follows.

\section{CASE WHERE $\ell=p$ OR $\ell=q$}

Throughout this section, we set $P:=p$ if $p \geq 5$; and $P:=9$ if $p=3$. Suppose that

$$
\ell=p \quad \text { and }\left\{\begin{array}{l}
\text { either } q \not \equiv 1(\bmod P) \text { or } \\
q \equiv 1(\bmod P) \text { and } p^{\frac{q-1}{p}} \not \equiv 1(\bmod q) .
\end{array}\right.
$$

Theorem 4.1. We have $\mathcal{T}\left[p^{\infty}\right]=\mathcal{C}\left[p^{\infty}\right]$.

Proof. We divide the problem into three cases.

(1) Suppose that $q \not \equiv 1(\bmod P)$ and $q \equiv 1(\bmod p)$. This happens when $\ell=p=3$. In this case, the indices of $\mathcal{I}_{1}, \mathcal{I}_{2}$ and $\mathcal{I}_{3}$ are not divisible by 3 (cf. [9, Theorem 1.4]). Therefore there are no Eisenstein maximal ideals containing 3 and $\mathbb{T}_{p} / \mathcal{I}_{0}=0$. Thus, we have $\mathcal{T}\left[3^{\infty}\right]=\mathcal{C}\left[3^{\infty}\right]=0$.

(2) Suppose that $q \equiv 1(\bmod P)$ and $p^{\frac{q-1}{p}} \not \equiv 1(\bmod q)$. Then, $\mathfrak{m}_{1}=\mathfrak{m}_{2}$ is not new by [8, Theorem 3.1]. Since $U_{p} \equiv p \equiv 0\left(\bmod \mathfrak{m}_{3}\right), \mathfrak{m}_{3}$ is not new. Therefore $\mathbb{T}_{p} / \mathcal{I}_{0} \simeq \mathbb{T}_{p}^{\text {old }} / \mathcal{I}_{0}$. Consider the following exact sequence:

$$
0 \longrightarrow J_{\text {old }}(\mathbb{Q})\left[p^{\infty}\right] \longrightarrow J(\mathbb{Q})\left[p^{\infty}\right] \longrightarrow J^{\text {new }}(\mathbb{Q})\left[p^{\infty}\right] .
$$

If $J^{\text {new }}(\mathbb{Q})\left[p^{\infty}\right] \neq 0$, then there is a new Eisenstein maximal ideal containing $p$, which is a contradiction. Therefore we have $J_{\text {old }}(\mathbb{Q})\left[p^{\infty}\right]=J(\mathbb{Q})\left[p^{\infty}\right]$. Now, the result follows from [1, Theorem 2] because $p$ does not divide $2(p-1, q-1)$.

(3) Suppose that $q \not \equiv 1(\bmod p)$. First, assume that $q \not \equiv-1(\bmod P)$. Then, the indices of $\mathcal{I}_{1}, \mathcal{I}_{2}$ and $\mathcal{I}_{3}$ are not divisible by $p$, there is no Eisenstein maximal ideal. Thus, $\mathbb{T}_{p} / \mathcal{I}_{0}=0$ and $\mathcal{T}\left[p^{\infty}\right]=\mathcal{C}\left[p^{\infty}\right]=0$.

Next, assume that $q \equiv-1(\bmod P)$. By the same reason as above, $\mathfrak{m}_{1}$ and $\mathfrak{m}_{3}$ are not maximal (but $\mathfrak{m}_{2}$ is). Note that $\mathfrak{m}_{2}$ is neither $p$-old nor $q$-old by Mazur. Therefore we get $\mathbb{T}_{2} / \mathcal{I}_{0} \simeq \mathbb{T}_{\mathfrak{m}_{2}}^{\text {new }} / \mathcal{I}_{0}$. Since $\left(U_{p}-1\right)\left(U_{p}+1\right)=\left(U_{q}-1\right)\left(U_{q}+1\right)=0$ in $\mathbb{T}^{\text {new }}$, we get $\mathbb{T}_{2} / \mathcal{I}_{0}=\mathbb{T}_{2} / \mathcal{I}_{2} \simeq \mathbb{T}_{p} / \mathcal{I}_{2}$ by $[8$, Proposition 2.3]. As in the proof of Theorem 3.1, we get

$$
\mathcal{T}\left[p^{\infty}\right]=\mathcal{T}\left[p^{\infty}\right]\left[\mathcal{I}_{2}\right]=\mathcal{C}\left[p^{\infty}\right]\left[\mathcal{I}_{2}\right]=\mathcal{C}\left[p^{\infty}\right]
$$

Remark 4.2. If $p>q$, then the assumption above holds and hence $\mathcal{T}\left[p^{\infty}\right]=\mathcal{C}\left[p^{\infty}\right]$. Since $\mathcal{C}\left[p^{\infty}\right]=0$, there are no rational torsion points of order $p$ on $J_{0}(p q)$. 


\section{REFERENCES}

[1] Seng-Kiat Chua and San Ling, On the rational cuspidal subgroup and the rational torsion points of $J_{0}(p q)$, Proc. Amer. Math. Soc., Vol 125, Number 8 (1997), 2255-2263.

[2] Vladimir Drinfeld, Two theorems on modular curves, Functional Anal. Appl. 7 (1973), 155-156.

[3] Yuri Manin, Parabolic points and zeta functions of modular curves (in Russian), Izv. Akad. Nauk SSSR Ser. Mat., 36, 19-66 (1972). Translation in Math USSR-Izv 6 (1972), 19-64.

[4] Barry Mazur, Modular curves and the Eisenstein Ideal, Publications Math. de l'I.H.É.S., tome 47 (1977), $33-186$.

[5] Andrew Ogg, Diophantine equations and modular forms, Bull. A.M.S., Vol. 81 (1975), 14-27.

[6] Masami Ohta, Eisenstein ideals and the rational torsion subgroups of modular Jacobian varieties II, Tokyo Journal of Math., Vol. 37, no. 2 (2014), 273-318.

[7] Hwajong Yoo, The index of an Eisenstein ideal of multiplicity one, submitted, available at http: / /arxiv.org/pdf/1311.5275.pdf(2014).

[8] Hwajong Yoo, Rational torsion points on Jacobians of Shimura curves, submitted, available at http://math.uni.1u/ yoo/Shimura.pdf (2015).

[9] Hwajong Yoo, On Eisenstein ideals and the cuspidal group of $J_{0}(N)$, to appear Israel Journal of Math., available at http://arxiv.org/pdf/1502.01571.pdf(2015).

Center for Geometry and Physics, Institute for Basic Science (IBS), Pohang, RePublic of Korea 37673

E-mail address: hwajong@gmail.com 